\title{
Contraceptive Care in the Veterans Health Administration
}

\author{
Emmanuelle Yecies, MD ${ }^{1,2}$, Colleen Judge-Golden ${ }^{3}$, Lisa Callegari, MD, MPH ${ }^{4,5,6}$, Sonya \\ Borrero, MD, MS ${ }^{1,2,7}$ \\ ${ }^{1}$ Department of General Internal Medicine, University of Pittsburgh School of Medicine, \\ Pittsburgh, PA \\ ${ }^{2}$ VA Pittsburgh Healthcare System, Pittsburgh, PA \\ ${ }^{3}$ University of Pittsburgh School of Medicine, Pittsburgh, PA \\ ${ }^{4}$ Seattle-Denver Center of Innovation for Veteran-Centered and Value-Drive Care, VA Health \\ Services Research and Development, Seattle, WA \\ ${ }^{5}$ Department of Obstetrics \& Gynecology, University of Washington School of Medicine, Seattle, \\ WA \\ ${ }^{6}$ Department of Health Services, University of Washington School of Public Health, Seattle, WA \\ ${ }^{7}$ Center for Health Equity Research and Promotion, VA Pittsburgh Health Care System, \\ Pittsburgh, PA
}

\begin{abstract}
In recent years, the number of women Veterans obtaining care in the Veterans Affairs (VA) Healthcare System has grown, expanding the need for provision of contraceptive care. Women Veterans are a diverse group of women with complex sociodemographic and medical backgrounds, and meeting their needs presents a unique challenge for VA. Efforts including the establishment of comprehensive women's health clinics and training practitioners in women's health have greatly improved healthcare services for women Veterans over the last few decades. Recent data from a large cross-sectional survey study suggest that contraceptive use in VA is similar to the general population and that rates of unintended pregnancy, while still significant, are not higher than the general population. Subgroup analyses of this survey data, however, suggest that ongoing efforts are needed to improve outcomes in vulnerable subpopulations of women Veterans, particularly ethnic/racial minorities and Veterans with complex medical backgrounds. Policy changes such as the elimination of co-payments for contraceptive prescriptions and the dispensing of more months of contraceptive supply are evidence-based starting points for improvements, in addition to leveraging VA's integrated system and research infrastructure to improve patient-centered counseling and contraceptive access.
\end{abstract}

Corresponding Author: Emmanuelle Yecies; Address: 200 Lothrop Street, UPMC Montefiore Hospital, N715, Pittsburgh, PA 15213; Phone: 412-802-6579; yeciese@upmc.edu.

The authors report no conflict of interest.

The views expressed in this manuscript are those of the authors and do not necessarily reflect the position or policy of the VA. 


\section{Introduction}

Contraceptive counseling and provision is an integral component of primary care for women (1). Contraceptive care helps women prevent undesired pregnancies and space pregnancies to meet their reproductive goals, needs, and desires throughout their reproductive years. As the number of women Veterans of reproductive age seeking care in the Veterans Affairs (VA) Healthcare System continues to grow, VA's capacity to provide high-quality reproductive health services, including contraceptive care, is a high priority (2).

Women Veterans are a unique and vulnerable population, with disproportionate representation from racial/ethnic minority groups, high prevalence of medical co-morbidities and mental illness, and high rates of intimate partner violence (IPV) and histories of sexual assault compared to the general US population (3). Studies in non-Veteran populations indicate that these patient-level factors are important correlates of contraceptive use and unintended pregnancy. For example, compared to White women, women belonging to racial/ ethnic minority groups are less likely to use contraception overall and are more likely to use less effective methods (4-9). Black and Hispanic women also report lower contraceptive continuation and higher failure rates, and the unintended pregnancy rates in these minority groups remain significantly higher than in White women (10-14). Additionally, various medical and psychiatric co-morbidities, such as systemic lupus erythematosus and epilepsy, have been associated with lower contraceptive use and higher rates of unintended pregnancy in the general population (15-17). Finally, a history of emotional, physical or sexual abuse is also associated with contraceptive use, with differences in types of methods selected and higher rates of discontinuation in women who have experienced any form of abuse (18). The underlying causes of associations between these social and health factors and contraceptive use patterns are not yet completely understood, but could include differential access to healthcare and to the full range of contraceptive methods, knowledge and attitudes about contraception and pregnancy, and/or interactions with the healthcare system.

In this review, we first discuss current contraceptive services in the VA, including available methods, costs, and service delivery models. We then review the existing literature regarding contraceptive use patterns and unintended pregnancy among women Veterans who use the VA, including patient-, provider- and system-level correlates of use. We focus in particular on vulnerable populations of Veterans, including women of minority race/ethnicity and women with mental health and chronic medical disorders. Finally, we suggest directions for future research as well as potential policy changes to augment the quality and equity of contraceptive care within VA.

\section{Contraceptive Services in VA}

\section{Available Methods}

VA offers a variety of contraceptive methods which reflect the full range of FDA-approved contraceptive options. Prescription methods include moderately effective, short-acting methods (pill, patch, ring, and medroxyprogesterone injections) and highly effective permanent (sterilization) or long-acting reversible contraception (LARC), such as intrauterine devices (IUDs) and implants. Emergency contraception is also available. VA 
pharmacy services are required to adhere to a national formulary, a listing of products (drugs and supplies) determined to have "the highest quality and best value" (19). Abortion care is excluded with no exceptions, (see 38 CFR § 17.38(c)(1)), unlike the Department of Defense policy which allows for exceptions in cases of rape, incest, or life endangerment under the Shaheen amendment of the National Defense Authorization Bill.

\section{Method Costs}

Whether or not a Veteran incurs copayments for medications in VA is determined by a variety of factors, including financial means, timeframe of military service, and serviceconnection. Service connection describes a categorization in which Veterans receive additional benefits for injuries or illnesses that were incurred or aggravated during active military service $(20,21)$. Women Veterans who are not exempt from medication copays pay a flat fee for all contraception medications (the 2018 copay is $\$ 8$ for a 30 day supply). Contraceptive devices such as IUDs and implants do not have a copay beyond any cost associated with the insertion visit or follow-up visits. Similar to prescription copayments, visit copayments may also be waived or adjusted based on financial means and eligibility. Thus, women who use VA for primary care largely have access to low-cost or no-cost contraception. Copayments may represent a barrier to contraceptive use for some patients, however, as a retrospective cohort study of 3622 women Veterans from VA facilities in Southern California and Nevada found that copayments were significantly associated with reduced contraceptive adherence across all income levels (22).

\section{Service Delivery Models}

Women Veterans who receive primary care within VA are required to have access to care from a designated Women's Health Primary Care Provider (WH-PCP). Providers are designated as WH-PCPs based on completing and maintaining additional training in genderspecific care competencies, including contraceptive counseling and provision (23). While WH-PCPs are trained to provide the majority of counseling about contraception, gynecologists are available for more complex cases as well as methods that require insertion procedures, such as intrauterine devices and subdermal implants (24). Depending on the site, gynecology care can be accessed on site, at nearby VA sites, or with contracted community providers.

Primary care is generally provided in hospital-based clinics within VA Medical Centers (VAMCs) or in community-based outpatient clinics (CBOCs). The specific structure of women's primary care spaces typically falls into one of three models: (1) embedded in general primary care clinics, (2) separate from primary care but located within or adjacent to primary care clinic areas, and (3) in a completely separate women's health clinic (WHC), often with its own entrance and waiting room, which also often provide co-located additional services including pharmacy, social work, mental health and gynecology (23). Further details regarding the structure of women's healthcare in VA can be found in VA Handbook 1330.01, a comprehensive document establishing national guidelines for provision of health care to women Veterans (23). 
Understanding and tracking the structure of women's health care delivery is essential, as models of care have been associated with contraceptive service provision. In a study examining administrative data for 103,950 women Veterans who visited a primary care clinic in 2008, Borrero et al. demonstrated that women who were seen in a WHC were twice as likely to have a documented contraceptive method as those seen in a conventional primary care clinic (31\% v. 15\%) (25). While 97\% of all surveyed VA facilities prescribe and manage hormonal contraception, only $63 \%$ offer IUD placement (26); the odds of availability of onsite IUD placement are 20 times as high with the presence of an onsite gynecologist compared to clinics with no onsite gynecologist. In addition, hospital-based practices are significantly more likely to be able to place IUDs onsite compared to community-based practice (26). Finally, in a 2007 survey of 193 metropolitan VA sites, 50\% offered on-site IUD placement, but urban CBOCs were significantly less likely to offer onsite IUD placement compared to urban hospital-based clinics ( $28 \%$ vs $66 \%$ of sites, respectively) (27).

\section{Contraceptive use}

\section{Overall contraceptive use and method types}

The earliest studies regarding contraceptive use among women Veterans relied on VA administrative data. Borrero et al. examined VA administrative data of 103,950 reproductiveaged women Veterans who visited a primary care clinic in fiscal year 2008 and found that only $22 \%$ had a documented method of contraception at any point during the year (25).

Concerns about the limitations of administrative data in capturing contraceptive use (28), in particular use of methods that are over-the-counter or not obtained within VA, prompted VA researchers to survey women Veterans directly about contraceptive use and other reproductive health outcomes, such as unintended pregnancy. The Examining Contraceptive Use and Unmet Need among women Veterans (ECUUN) study (24), conducted by Borrero et al. between 2014 to 2016, surveyed reproductive-aged women Veterans across all regions and Veterans Integrated Service Networks (VISNs) in the US to assess contraceptive use, pregnancy history, and experiences with VA reproductive healthcare. Data were collected from 2302 women Veterans of reproductive age who had a primary care visit within VA in the prior 12 months. In this national sample, $62.3 \%$ of women reported using contraception in the month prior to their interview, suggesting much higher rates than previously detected from administrative data. Of those, $34.4 \%$ were using highly effective methods (male or female sterilization, IUD or implant), $17.4 \%$ were using moderately effective methods (contraceptive pills, patch, ring, or injection), and $10.2 \%$ were using least effective (nonprescription) methods (including condoms, natural family planning, and withdrawal).

Among the subset of women at risk for unintended pregnancy (defined as sexually active with a man in the prior 3 months; no history hysterectomy or infertility; and not pregnant, postpartum or seeking pregnancy), over a quarter $(27.4 \%)$ were found to have potential unmet need for contraception, defined as either using a non-prescription (least effective) contraceptive method (15.9\%) or not using contraception at all (11.5\%). Figure 1 shows contraceptive method use among women Veterans at risk for unintended pregnancy, as compared to women in an age-matched US population cohort. Rates of contraceptive use 
and unmet need for contraception were similar overall, although twice as many women Veterans at risk for unintended pregnancy were using LARC methods compared to the agematched U.S. population (23\% vs $11 \%$ ) (24). Over $65 \%$ of women received their LARC method within VA (29), suggesting that VA has made great strides in providing access to this form of contraception. Nevertheless, the high proportion of unmet contraceptive need suggests that women Veterans are likely to benefit from continued improvements in contraceptive care delivery.

\section{Vulnerable Populations}

The ECUUN study also provided important data on contraceptive use in vulnerable populations, with a particular focus on women of color and those with mental health disorders and medical illness. Women Veterans belonging to racial and ethnic minority groups had higher potential unmet need for contraception, with nearly $35 \%$ of Hispanic women and over $30 \%$ of non-Hispanic Black women with unmet need for prescription contraception, compared to $23 \%$ of non-Hispanic White women ( $\mathrm{p}<.001)$; racial/ethnic differences persisted even after adjusting for age, marital status, education, and income (24).

Racial/ethnic variation in contraceptive use may reflect a variety of factors, including differences in contraceptive and pregnancy preferences and knowledge, self-efficacy, relationship dynamics, and interactions with the healthcare system. Among the ECUUN cohort, Rosenfeld et al. identified disparities in contraceptive knowledge in different racial and ethnic groups, with both Black and Hispanic women scoring significantly lower on a range of contraception knowledge items than White women (30). In a subsequent study of the association of knowledge with contraceptive use, Callegari et. al. found that women with lower knowledge were less likely to use LARC methods, after adjusting for preferences and other potential confounders (31). Lower knowledge among racial/ethnic minority women may therefore contribute to observed differences in method selection.

Callegari et al. examined variations by race/ethnicity in contraceptive preferences, beliefs and self-efficacy among women Veterans that could also drive some of the observed differences in method use (32). Controlling for age, marital status, education, income, religion, parity, deployment history, and history of medical and mental health conditions, Black women had lower odds of considering method effectiveness extremely important compared to White women, while also having higher odds of rating as extremely important that the method "does not contain any hormones" and "prevents sexually transmitted infections" (32). Hispanic and Black women also had higher odds of expressing fatalistic beliefs about pregnancy than White women and of viewing contraception as primarily a woman's responsibility (32). Finally, self-efficacy was lower in both Hispanic and Black women compared to White women, with lower odds of being very sure that they could use a contraceptive method as indicated for a year.

In the study examining associations of preferences with contraceptive use, women who expressed a preference that a method is highly effective were more likely to use moderately effective and highly effective methods compared to less effective, non-prescription methods or no method. Women who expressed a preference for a method not containing hormones were less likely to use methods containing hormones. Women who expressed low self- 
efficacy were less likely to use moderately or highly effective methods (31). Variations in preferences and attitudes by race/ethnicity may help to explain differences in contraceptive use and type of method selected.

Relationship dynamics may also contribute to differential contraceptive use by race/ ethnicity. Rosenfeld et al. found that younger, single and Black women Veterans were more likely to report recent reproductive coercion, or a male partner's attempts to promote pregnancy by interfering with her contraceptive use or reproductive decision making. Reproductive coercion was in turn associated with reductions in overall contraceptive use and prescription contraceptive use at last sexual intercourse (33). Interactions with the health care system may also differentially influence women's contraceptive choices. MacDonald et al. found that women who reported experiencing perceived race-based discrimination when receiving VA care were less likely to use prescription contraceptive methods than were women who did not perceive discrimination. Differences in use were largest for LARC methods. As expected, perceived race-based discrimination was reported by higher proportions of Black and Hispanic women compared to White women $(11.3 \%$ and $11.2 \%$ vs. $4.4 \%$, respectively; $\mathrm{p}<0.001$ ) (34). These findings underscore the fact that racial differences in contraceptive use are driven by a variety of variables, many of which clinicians may be able to elicit during nuanced counseling in order to improve the provision of contraceptive care and ensure that method selection is the result of informed and preference-concordant decision making.

Mental illness has also been found to affect contraceptive use. An early study used administrative data to examine mental illness (depression, posttraumatic stress disorder, anxiety, bipolar disorder, schizophrenia and adjustment disorder), substance use disorder (SUD) and contraceptive use among 94,115 reproductive aged women Veterans seen in VA primary care in 2008 (35). Overall, $22.1 \%$ of women with mental illness without SUD (36.5\% of the study population) had documentation of a contraceptive method, compared to $17.7 \%$ of women without mental illness or SUD. Women with mental illness were also significantly more likely to be using LARC methods compared to women Veterans without mental illness. However, women with SUD with or without a comorbid mental illness (5.9\% of the study population) were significantly less likely to be using prescription contraceptive methods than women with neither diagnosis. In a more recent study using survey data from ECUUN, women Veterans with self-reported mental illness diagnoses (including major depression, bipolar disorder, post-traumatic stress disorder, schizophrenia, and anxiety/panic disorder) were found to have similar rates of contraceptive use and method efficacy at last sex compared to Veterans without a diagnosis of mental illness (36). These two studies together do not suggest that mental health diagnoses are a barrier to contraceptive access in the VA, which may reflect the strong integration of mental health services in primary care within the VA system. Questions remain regarding contraception for women with SUD, in part due to the limitations associated with identifying SUD with administrative data (the ECUUN study did not assess SUD).

Nearly one third (29.0\%) of women Veterans at risk for unintended pregnancy in the ECUUN study reported at least one medical contraindication to estrogen-containing hormonal contraception, most commonly hypertension (14.9\%), migraines with aura (8.7\%) 
or being a smoker over age 35 (7.4\%) (37). Reassuringly, these women Veterans were less likely to report current use of estrogen-containing contraceptive methods compared to women without contraindications $(13.6 \%$ vs. $25.5 \%$, p<0.001). However, women with contraindications were also significantly more likely to report no method of contraception compared to those with no medical contraindications ( $14.8 \%$ vs. $10.2 \%, \mathrm{p}=0.03)$, identifying potential unmet need in this medically at-risk population (37). Further research is needed to elucidate the cause of this finding, and to determine whether efforts to improve contraceptive use among women with medical contraindications should focus on provider education, patient access to contraception, facilitating more nuanced contraceptive counseling, or other strategies.

Beyond estrogen-contraindicating conditions, contraceptive use in women with medical illness is of particular relevance, as prior research found that $49 \%$ of female Veterans who filled a prescription received a potentially teratogenic prescription medication from VA pharmacies (38). In this study of 2,634,441 prescriptions filled in FY 2008 by 78,232 reproductive-aged veterans, Schwarz et al. found that only $37.3 \%$ of women Veterans receiving class $\mathrm{D}$ or $\mathrm{X}$ prescriptions had documentation of family planning counseling, and only $32.6 \%$ had documentation of a contraceptive method. Despite the limitations of this data, including reliance on electronic records as the sole source of information, these findings suggest the need for interventions to improve the contraceptive care of women Veterans with medical illness, particularly those using teratogenic medications.

Finally, studies using administrative data indicate that a history of military sexual trauma (MST) is associated with contraceptive use. MST refers to sexual assault or repeated threatening sexual harassment while a Veteran was in the military, and a comprehensive screening program exists to identify MST in Veterans (39). A retrospective cohort study that included 68,466 women Veterans aged 18-45 years who served in Operation Enduring Freedom and Operation Iraqi Freedom (OEF/OIF) and presented to a VA primary care clinic between 2002 and 2010 demonstrated that women Veterans reporting MST had increased odds of receiving prescription contraception compared to those who denied a history of MST (40). The authors also found that women reporting MST were more likely than those without MST to receive care from a women's health clinic, which may explain the increased provision of effective prescription contraceptive methods.

Taken together, these studies demonstrate that overall contraceptive use is similar among Veteran and non-Veteran populations, although Veterans appear to use LARC methods at higher rates. These studies also indicate that certain groups of women Veterans, including women of color and those with medical conditions and substance use disorders, may have ongoing unmet contraceptive need. By attending to issues around knowledge, preferences and contraindications, and identifying how these factors influence contraception use and method selection, clinicians can begin to build a robust counseling framework in their practice and provide high-quality, patient-centered contraceptive care.

\section{Contraceptive Adherence}

In addition to method type, method adherence and continuation are important determinants of contraceptive efficacy and women's risk of unintended pregnancy. To date, studies on 
adherence to contraception among women Veterans have used administrative data and focused on patterns of medication possession and refills across racial/ethnic groups and mental illness status.

In a 2013 study examining data from 6,946 reproductive age women Veterans who received short-acting contraception in FY2008, 64\% of women experienced at least one gap in contraceptive coverage of 7 days or longer, and only $22 \%$ received 12 full months of contraception without gaps over the course of one year (41). There were significant differences by race/ethnicity, with Hispanic and Black women receiving fewer months of contraceptive supply on average over the course of a year compared to non-Hispanic White women $(8.9,9.0$, and 9.3 months, respectively; $\mathrm{p}<0.001)$. Hispanic Veterans were also more likely to experience gaps in contraceptive coverage compared to their White counterparts (70\% v. 64\%, respectively; $\mathrm{p}=0.02$ ). Of note, women who received 3 month supplies compared to 1 month supplies experienced fewer gaps and improved overall coverage, suggesting that prescribing and dispensing more months of contraception may be a successful strategy to improve contraception care (41).

In another study using administrative data from 9,780 women using hormonal contraceptive methods in FY13, women Veterans with a history of mental illness with or without coexisting substance abuse had similar occurrence of gaps in contraceptive coverage, but gaps were significantly longer compared to women without mental illness (42). As a result, women with mental illness (with or without SUD) had significantly fewer months of contraceptive coverage compared to those without any diagnoses (7.7 and 8.2 vs. 8.5 months, respectively; $\mathrm{p}<0.001)$. These studies suggest that there are opportunities to enhance contraceptive adherence and continuation with the VA.

\section{Unintended pregnancy}

A key benefit of contraception is allowing women to time and space their pregnancies in a way that best fits their needs. While there is increasing recognition that unintended pregnancy is not a universally negative outcome $(43,44)$, population rates of unintended pregnancy remain a standard benchmark or indicator of successful contraceptive care. In order to understand ongoing gaps in contraception care at the VA, it is therefore paramount to examine unintended pregnancy rates.

In the ECUUN study, the rate of unintended pregnancy, defined as pregnancies reported as either "unwanted" (i.e. no future pregnancies were desired) or occurring "sooner than desired," was found to be 26.1 per 1000 women (95\% CI 19.6-32.6), as compared to 34.4 in the age-matched US population (24). This seemingly lower rate of unintended pregnancy is largely reflective of lower overall rates of pregnancy, as the proportion of pregnancies that were unintended is similar to the general population (37.1\%, 95\% CI: 29.6-44.3 compared to $35.2 \%$ ). Although differences in rates of UIP among subpopulations have not been thoroughly examined in Veteran populations, one study found associations between mental illness and history of unintended pregnancy among women Veterans. Women Veterans with any mental health disorder were more likely to report a history of any unintended pregnancy 
and had experienced greater numbers of unintended pregnancies compared to women with no mental illness (24).

Although VA does not provide abortion care, the rate of pregnancy termination in the past five years was similar between women Veterans and U.S. women, and women Veterans were slightly more likely to report ever having had an abortion $(17.7 \%, 95 \%$ CI $16.1 \%-19.3 \% \mathrm{v}$. $15.2 \%$ ) (45). Although under-reporting is a concern with all studies on abortion rates, differential under-reporting between Veterans and non-Veterans is unlikely. In all, it appears that women Veterans have similar rates of unintended pregnancy and abortion as the general population.

\section{Conclusions and Future Directions}

Women Veterans are a rapidly growing population within the VA healthcare system, necessitating ongoing efforts to optimize gender-specific care provision such as contraceptive care. Women Veterans are disproportionately from minority racial/ethnic groups and have a high prevalence of mental health issues and medical co-morbid conditions, all of which are characteristics that have been linked to possible unmet contraceptive need and/or risk of unintended pregnancy in non-VA populations. VA-specific data discussed in this review has confirmed that these factors are associated with differences in use and adherence to contraception, even in a healthcare setting in which women should have uniform access to low-cost or no-cost contraception.

To ensure that high-quality contraceptive care is available to women Veterans, VA has developed extensive and comprehensive models of care, utilizing designated clinics and providers. Data demonstrates that women Veterans have similar rates of contraceptive use and unintended pregnancy compared to the general population. In fact, women Veterans use highly effective LARC methods at higher rates than the general population. However, disparities in knowledge, contraceptive method selection and adherence, and unmet contraceptive need persist among women in vulnerable populations.

Characteristics such as race/ethnicity and co-morbid medical and mental health conditions may serve as proxies for modifiable differences in factors such as knowledge, access, and experiences, including perceived race-based discrimination, within the medical system. The differences in contraceptive use patterns suggest that individualized, patient-centered approaches that encourage discussion and are attentive to women's unique social, structural, and medical contexts are necessary to optimize care and outcomes in this population. Studies are underway to test interventions to enhance reproductive health services in this complicated population, including a study of a web-based decision support tool ("MyPath") to facilitate patient-centered communication about reproductive health needs and promote informed and value-concordant contraceptive decision making (46).

System and policy level interventions are also needed to target gaps in contraceptive care. System-level changes such as electronic health record prompts or reminders for routine assessment of women Veterans' reproductive goals and documentation of risk of unintended pregnancy could help ensure that reproductive-aged women Veterans receive recommended 
and indicated services, include safe medication prescribing. To help improve contraceptive adherence, contraception dispensing could be exempted from the usual 90-day supply maximum to allow for dispensing of 12-month supplies. Research in non-VA settings has shown that increasing the number of months of contraceptive supply to 12 months is associated with improved adherence and continuation (43), and this research has led to calls from multiple organizations including the American College of Obstetricians and Gynecologists and the Centers for Disease Control and Prevention to dispense up to a year of contraception at a time $(47,48)$. Such a strategy may also help address adherence disparities in VA. Another potential avenue for policy change is the elimination of copays for contraception. In the private sector, the Affordable Care Act has eliminated co-pays and cost-sharing for contraception for most US women. To date, VA has not adopted this policy, but given data that copays are associated with reduced contraceptive adherence in the Veteran population (22), eliminating co-pays for contraception could also improve access and adherence and reduce unintended pregnancies. Finally, VA policies around abortion are more restrictive than the Department of Defense (DoD), which allows for abortions in cases of rape, incest or when the life of the mother is threatened. Expanding access to abortion in these situations would not only create equity with DoD policy but would provide a critical reproductive health service for women.

In summary, data demonstrate important strides in contraceptive care and services for women Veterans while highlighting opportunities for improvement. VA research, operations and policy efforts should continue to strive towards optimizing high-quality, equitable contraceptive care for the diverse population of women it serves, particularly as this population continues to grow.

\section{Acknowledgments}

Colleen Judge-Golden is supported by the National Center For Advancing Translational Sciences of the National Institutes of Health under Award Number TL1TR001858 (PI: Wishwa Kapoor). Lisa Callegari is supported by a VA Health Services Research and Development (HSR\&D) Career Development Award (CDA-14-412).

\section{References}

1. Women IoMCoPSf, Health BoP, Practice PH. Clinical preventive services for women: Closing the gaps: National Academies Press; 2011.

2. Zephyrin LC, Katon JG, Yano EM. Strategies for transforming reproductive healthcare delivery in an integrated healthcare system: a national model with system-wide implications. Current opinion in obstetrics \& gynecology. 2014;26(6):503-10. [PubMed: 25333678]

3. Frayne SM, Phibbs CS, Saechao F, Maisel NC, Friedman SA, Finlay A, et al. Sourcebook: Women Veterans in the Veterans Health Administration Volume 3 Sociodemographics, Utilization, Costs of Care, and Health Profile. Women's Health Evaluation Initiative, Women's Health Services, Veterans Health Administration, Department of Veterans Affairs, Washington DC 22014.

4. Krings KM, Matteson KA, Allsworth JE, Mathias E, Peipert JF. Contraceptive choice: how do oral contraceptive users differ from condom users and women who use no contraception? American journal of obstetrics and gynecology. 2008;198(5):e46-7. [PubMed: 18313637]

5. Dehlendorf C, Park SY, Emeremni CA, Comer D, Vincett K, Borrero S. Racial/ethnic disparities in contraceptive use: variation by age and women's reproductive experiences. American journal of obstetrics and gynecology. 2014;210(6):526.e1-9. [PubMed: 24495671]

6. Mosher W, Jones J, Abma J. Nonuse of contraception among women at risk of unintended pregnancy in the United States. Contraception. 2015;92(2):170-6. [PubMed: 25998937] 
7. Daniels K, Daugherty J, Jones J, Mosher W. Current Contraceptive Use and Variation by Selected Characteristics Among Women Aged 15-44: United States, 2011-2013. National health statistics reports. 2015(86):1-14.

8. Frost JJ, Singh S, Finer LB. Factors associated with contraceptive use and nonuse, United States, 2004. Perspectives on sexual and reproductive health. 2007;39(2):90-9. [PubMed: 17565622]

9. Shih G, Vittinghoff E, Steinauer J, Dehlendorf C. Racial and ethnic disparities in contraceptive method choice in California. Perspectives on sexual and reproductive health. 2011;43(3):173-80. [PubMed: 21884385]

10. Frost JJ, Darroch JE. Factors associated with contraceptive choice and inconsistent method use, United States, 2004. Perspectives on sexual and reproductive health. 2008;40(2):94-104. [PubMed: 18577142]

11. Rocca CH, Harper CC. Do racial and ethnic differences in contraceptive attitudes and knowledge explain disparities in method use? Perspectives on sexual and reproductive health. 2012;44(3):150-8. [PubMed: 22958659]

12. Kost K, Singh S, Vaughan B, Trussell J, Bankole A. Estimates of contraceptive failure from the 2002 National Survey of Family Growth. Contraception. 2008;77(1):10-21. [PubMed: 18082661]

13. Vaughan B, Trussell J, Kost K, Singh S, Jones R. Discontinuation and resumption of contraceptive use: results from the 2002 National Survey of Family Growth. Contraception. 2008;78(4):271-83. [PubMed: 18847574]

14. Finer LB, Zolna MR. Declines in Unintended Pregnancy in the United States, 2008-2011. The New England journal of medicine. 2016;374(9):843-52. [PubMed: 26962904]

15. Davis AR, Pack AM, Kritzer J, Yoon A, Camus A. Reproductive history, sexual behavior and use of contraception in women with epilepsy. Contraception. 2008;77(6):405-9. [PubMed: 18477488]

16. Schwarz EB, Manzi S. Risk of unintended pregnancy among women with systemic lupus erythematosus. Arthritis and rheumatism. 2008;59(6):863-6. [PubMed: 18512717]

17. Ozcan NK, Boyacioglu NE, Enginkaya S, Dinc H, Bilgin H. Reproductive health in women with serious mental illnesses. Journal of clinical nursing. 2014;23(9-10):1283-91. [PubMed: 24720577]

18. Allsworth JE, Secura GM, Zhao Q, Madden T, Peipert JF. The impact of emotional, physical, and sexual abuse on contraceptive method selection and discontinuation. American journal of public health. 2013;103(10):1857-64. [PubMed: 23948012]

19. MJ K. VHA HANDBOOK 1108.082009.

20. Returning Servicemembers (OEF/OIF/OND) [Available from: https://www.va.gov/healthbenefits/ apply/returning_servicemembers.asp.

21. Affairs USDoV. Health Benefits Copays 2018 [Available from: https://www.va.gov/healthbenefits/ cost/copays.asp.

22. Kazerooni R, Vu K, Takizawa A, Broadhead C, Morreale AP. Association of copayment and socioeconomic status with hormonal contraceptive adherence in a female veteran population. Women's health issues : official publication of the Jacobs Institute of Women's Health. 2014;24(2):e237-41.

23. DJ S. VHA DIRECTIVE 1330.01. 2017.

24. Borrero S, Callegari LS, Zhao X, Mor MK, Sileanu FE, Switzer G, et al. Unintended Pregnancy and Contraceptive Use Among Women Veterans: The ECUUN Study. Journal of general internal medicine. 2017;32(8):900-8. [PubMed: 28432564]

25. Borrero S, Mor MK, Zhao X, McNeil M, Ibrahim S, Hayes P. Contraceptive care in the VA health care system. Contraception. 2012;85(6):580-8. [PubMed: 22176794]

26. Cope JR, Yano EM, Lee ML, Washington DL. Determinants of contraceptive availability at medical facilities in the Department of Veterans Affairs. Journal of general internal medicine. 2006;21 Suppl 3:S33-9. [PubMed: 16637943]

27. Katon J, Reiber G, Rose D, Bean-Mayberry B, Zephyrin L, Washington DL, et al. VA location and structural factors associated with on-site availability of reproductive health services. Journal of general internal medicine. 2013;28 Suppl 2:S591-7. [PubMed: 23807070] 
28. Womack JA, Scotch M, Leung SN, Skanderson M, Bathulapalli H, Haskell SG, et al. Use of structured and unstructured data to identify contraceptive use in women veterans. Perspectives in health information management. 2013;10:1e.

29. Koenig AF, Zhao X, Callegari LS, Mor M, Borrero S. Long-acting reversible contraceptive use among women veterans. Contraception. 2017;96(4):267.

30. Rosenfeld E, Callegari LS, Sileanu FE, Zhao X, Schwarz EB, Mor MK, et al. Racial and ethnic disparities in contraceptive knowledge among women veterans in the ECUUN study.

Contraception. 2017;96(1):54-61. [PubMed: 28322769]

31. Callegari LSRE, Zhao X, Schwarz EB, Mor MK, Borrero S. Relationships between contraceptive preferences, knowledge and contraceptive use among women veterans.

32. Callegari LS, Zhao X, Schwarz EB, Rosenfeld E, Mor MK, Borrero S. Racial/ethnic differences in contraceptive preferences, beliefs, and self-efficacy among women veterans. American journal of obstetrics and gynecology. 2017;216(5):504.e1-.e10. [PubMed: 28063910]

33. Rosenfeld EA, Miller E, Zhao X, Sileanu FE, Mor MK, Borrero S. Male partner reproductive coercion among women veterans. American journal of obstetrics and gynecology. 2018;218(2):239.e1-.e8. [PubMed: 29056537]

34. MacDonald S, Hausmann LRM, Sileanu FE, Zhao X, Mor MK, Borrero S. Associations Between Perceived Race-based Discrimination and Contraceptive Use Among Women Veterans in the ECUUN Study. Medical care. 2017;55 Suppl 9 Suppl 2:S43-s9. [PubMed: 28806365]

35. Callegari LS, Zhao X, Nelson KM, Lehavot K, Bradley KA, Borrero S. Associations of mental illness and substance use disorders with prescription contraception use among women veterans. Contraception. 2014;90(1):97-103. [PubMed: 24731860]

36. Judge-Golden CP, B S, Z X, M MK, C LS. The association between mental health disorders and history of unintended pregnancy among women Veterans. 2018.

37. Judge CP, Zhao X, Sileanu FE, Mor MK, Borrero S. Medical contraindications to estrogen and contraceptive use among women veterans. American journal of obstetrics and gynecology. 2018;218(2):234.e1-.e9. [PubMed: 29111146]

38. Schwarz EB, Longo LS, Zhao X, Stone RA, Cunningham F, Good CB. Provision of potentially teratogenic medications to female veterans of childbearing age. Medical care. 2010;48(9):834-42. [PubMed: 20706159]

39. Kimerling R, Gima K, Smith MW, Street A, Frayne SJAJoPH. The Veterans Health Administration and military sexual trauma. 2007;97(12):2160-6.

40. Goyal V, Mattocks K, Bimla Schwarz E, Borrero S, Skanderson M, Zephyrin L, et al. Contraceptive provision in the VA healthcare system to women who report military sexual trauma. Journal of women's health (2002). 2014;23(9):740-5.

41. Borrero S, Zhao X, Mor MK, Schwarz EB, Good CB, Gellad WF. Adherence to hormonal contraception among women veterans: differences by race/ethnicity and contraceptive supply. American journal of obstetrics and gynecology. 2013;209(2):103.e1-11. [PubMed: 23524170]

42. Callegari LS, Zhao X, Nelson KM, Borrero S. Contraceptive adherence among women Veterans with mental illness and substance use disorder. Contraception. 2015;91(5):386-92. [PubMed: 25636807]

43. Aiken AR, Borrero S, Callegari LS, Dehlendorf C. Rethinking the Pregnancy Planning Paradigm: Unintended Conceptions or Unrepresentative Concepts? Perspectives on sexual and reproductive health. 2016;48(3):147-51. [PubMed: 27513444]

44. Borrero S, Nikolajski C, Steinberg JR, Freedman L, Akers AY, Ibrahim S, et al. "It just happens": a qualitative study exploring low-income women's perspectives on pregnancy intention and planning. Contraception. 2015;91(2):150-6. [PubMed: 25477272]

45. Schwarz EB, Sileanu FE, Zhao X, Mor MK, Callegari LS, Borrero S. Induced abortion among women veterans: data from the ECUUN study. Contraception. 2018;97(1):41-7. [PubMed: 29055781]

46. Callegari LSMS, Tartaglione E, Nelson K, Arterburn DA, Zephyrin LZ, Borrero S. Development of a Web-Based Decision Support Tool to Promote Patient-Centered Family Planning Counseling in VA Primary Care. 
47. Committee opinion no. 615: Access to contraception. Obstetrics and gynecology. 2015;125(1):250-5. [PubMed: 25560140]

48. Curtis KM, Jatlaoui TC, Tepper NK, Zapata LB, Horton LG, Jamieson DJ, et al. U.S. Selected Practice Recommendations for Contraceptive Use, 2016. MMWR Recommendations and reports : Morbidity and mortality weekly report Recommendations and reports. 2016;65(4):1-66. 
Women Veterans ages 20-44 a

2014-2016

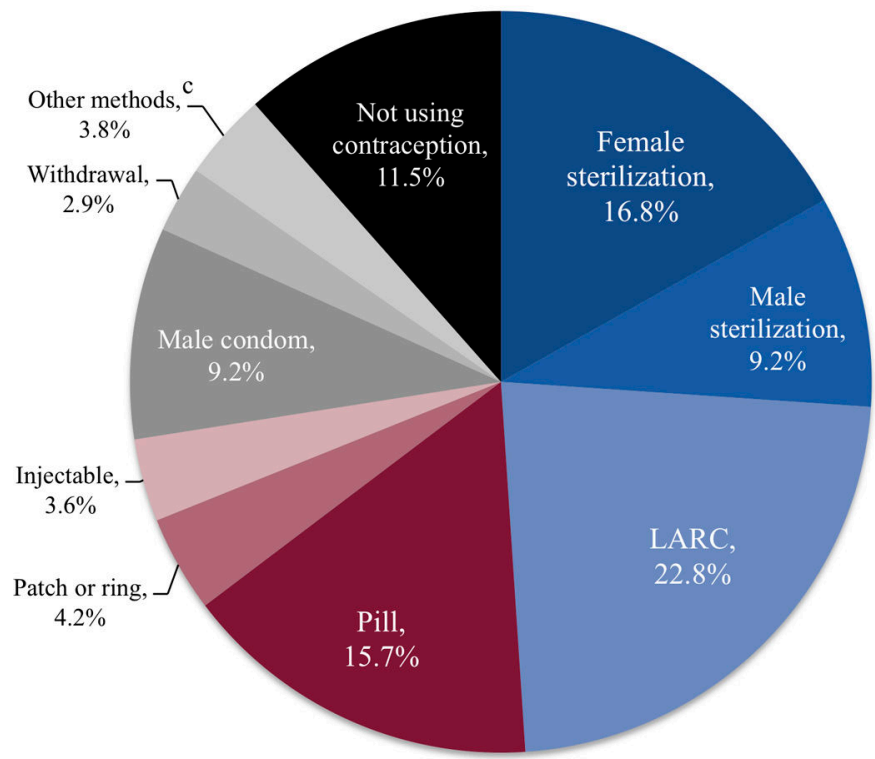

U.S. population ages $20-44^{\text {b }}$

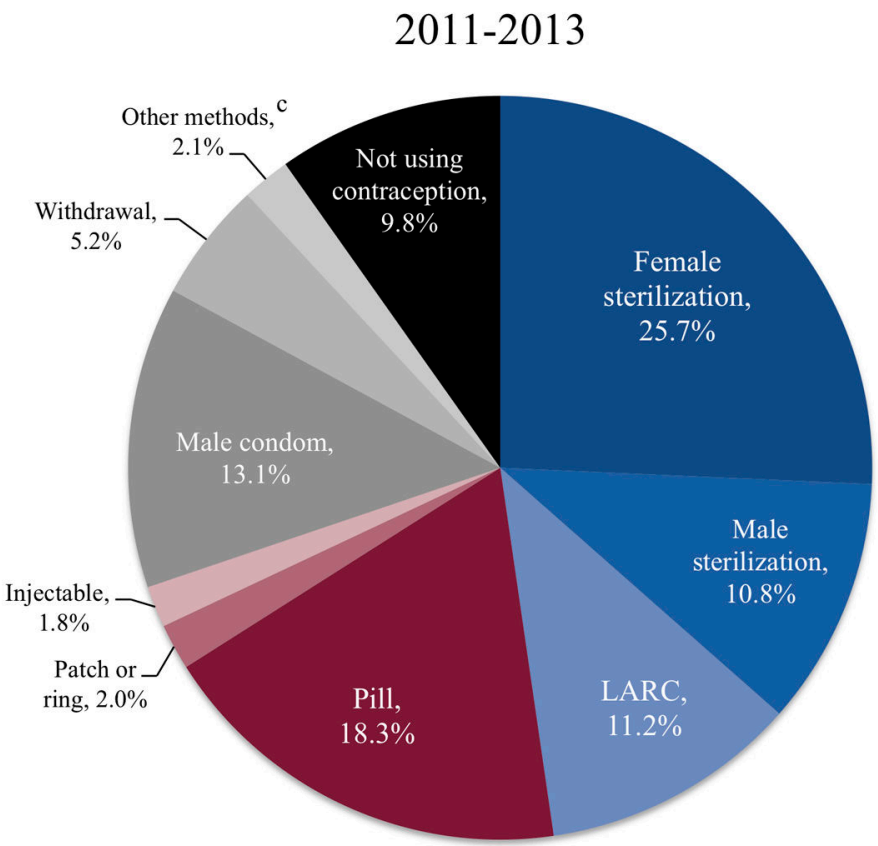

Figure 1. Contraceptive use among women Veterans and women in the general US population at risk for unintended pregnancy

Adapted from Borrero et al. 2017.

Women were considered at risk of unintended pregnancy if they had been sexually active with a man in the prior 3 months, had not had a hysterectomy, and were not infertile, pregnant, postpartum, or trying to get pregnant.

${ }^{\text {a }} \mathrm{N}=1173$. A total of 4 women $(0.3 \%)$ reported using contraception but did not specify method type and are thus not included in individual method reporting. b $\mathrm{N}=2648$. Age-specific contraception use estimates were obtained from the 2011-2013 National Survey of Family Growth data for women aged 20-44 with at least a high school education or GED and at risk of pregnancy and were applied to the VA population age distribution. Age was categorized by 5-year groups as follows: 20-24, 25- 29, 30-34, 3539 , and 40-44. The weighted sample size is 31,385,000.

c Other methods included spermicide, diaphragm, cervical cap or Today sponge, female condom, and emergency contraception. 